\title{
Identification of post-stroke EEG signal using wavelet and convolutional neural networks
}

\author{
Esmeralda C. Djamal ${ }^{1}$, Rizkia I. Ramadhan ${ }^{2}$, Miranti I. Mandasari ${ }^{3}$, Daswara Djajasasmita ${ }^{4}$ \\ ${ }^{1,2}$ Department of Informatics, Universitas Jenderal Achmad Yani, Indonesia \\ ${ }^{3}$ Faculty of Industrial Technology, Institut Teknologi Bandung, Indonesia \\ ${ }^{4}$ Faculty of Medicine, Universitas Jenderal Achmad Yani, Indonesia
}

\begin{tabular}{l} 
Article Info \\
\hline Article history: \\
Received Nov 3, 2019 \\
Revised Feb 3, 2020 \\
Accepted Mar 15, 2020 \\
\hline
\end{tabular}

\section{Keywords:}

Convolutional neural networks EEG signal

Extraction

Post-stroke

Wavelet

\begin{abstract}
Post-stroke patients need ongoing rehabilitation to restore dysfunction caused by an attack so that a monitoring device is required. EEG signals reflect electrical activity in the brain, which also informs the condition of post-stroke patient recovery. However, the EEG signal processing model needs to provide information on the post-stroke state. The development of deep learning allows it to be applied to the identification of post-stroke patients. This study proposed a method for identifying post-stroke patients using convolutional neural networks $(\mathrm{CNN})$. Wavelet is used for EEG signal information extraction as a feature of machine learning, which reflects the condition of post-stroke patients. This feature is Delta, Alpha, Beta, Theta, and Mu waves. Moreover, the five waves, amplitude features are also added according to the characteristics of the post-stroke EEG signal. The results showed that the feature configuration is essential as distinguish. The accuracy of the testing data was $90 \%$ with amplitude and Beta features compared to $70 \%$ without amplitude or Beta. The experimental results also showed that adaptive moment estimation (Adam) optimization model was more stable compared to Stochastic gradient descent (SGD). But SGD can provide higher accuracy than the Adam model.
\end{abstract}

This is an open access article under the CC BY-SA license.

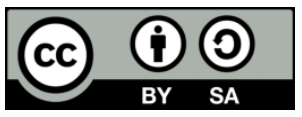

\section{Corresponding Author:}

Esmeralda C. Djamal,

Department of Informatics,

Universitas Jenderal Achmad Yani,

Jalan Terusan Jenderal Sudirman, Cimahi, Indonesia.

Email: esmeralda.contessa@lecture.unjani.ac.id

\section{INTRODUCTION}

Stroke more often leaves disability than death. Stroke is the third-largest cause of disability in the world [1]. It is the number one cause of death in Indonesia based on the results of research published by the Health Research and Development Agency (Balitbangkes) in 2015. Therefore, medical rehabilitation is essential to restore post-stroke patients in independence to take care of them and carry out daily life activities. In stroke rehabilitation, there is a standard clinical procedure, namely the National Institute of Health Stroke Scale (NIHSS), to determine the current condition of patients [2]. The NIHSS tool can be an evaluation of the rehabilitation of stroke patients. NIHSS uses 11 indicators for post-stroke patients, namely the level of consciousness, eye gaze, vision, facial paralysis, hand and foot movements, joint inflammation, sensory abilities, language disorders, speech disorders, and sensory abilities. In each indicator, there is an assessment of the score between 0-4. But some variable use range 0-2. Scoring informs five levels of stroke, namely, score 0 no indication of a stroke at all (no stroke), score 1-4 mild stroke (minor stroke), score 5-15 medium stroke (moderate stroke), score 16-20 medium stroke towards severe stroke, and 21-42 severe strokes [3]. 
Stroke can be detected by several instruments, such as CT scan, MRI, electroencephalogram (EEG), and EMG. CT scan and MRI are usually for the first treatment detection. The computational capabilities, automatically neurorehabilitation, can use EMG [4] and EEG [5]. EEG records reflect brain function. The instrument can provide supporting information in identifying and monitoring different neurological rehabilitation [5]. EEG has advantages in low cost and minimal risk to patients, but it is necessary to process to inform the brain's condition properly. Some studies used EEG signals to identify ischemic stroke patients [6], investigate that stroke patients are able to use BCI [7], and extracted significant variable [8].

EEG signal processing consists of two essential factors that are feature extraction and identification [9]. Usually, the neurologist reads the EEG signal of a post-stroke patient by observing wave density or rhythm, amplitude, asymmetric, change in magnitude, the presence of waves, and the ratio between waves. The most important thing is how to extract the signal into frequency components. The next step is to choose waves with the right frequency component under the characteristics of the EEG signal variable being reviewed [10]. Therefore, research using frequency extraction is beneficial. Several previous studies used Wavelet extraction to determine the significant variables of EEG signals for post-stroke patients [8]. Wavelet can extract Alpha, Beta, Theta, Gamma, and $\mathrm{Mu}$ waves to classify emotions of stroke patients [11] and healthy people [12]. Also, wavelet provides easy time-frequency analysis. Past research shows that the study of the time-frequency of stroke patients during TMS therapy [13]. Many other studies have also looked at the characteristics of waves with a specific frequency in stroke patients [14], which is one characteristic. Previous studies used Wavelet extraction of EEG signals to find significant variables of stroke [8] and the classification of emotions [15]. In meanwhile, FFT is suitable for identifying EEG signal asymmetries [16].

Machine learning allows computers to learn like humans make computers learn EEG stroke patterns previously so that automatic detection. Deep learning is part of machine learning, which in recent years has shown remarkable performance. Popular methods used in deep learning are convolutional neural network $(\mathrm{CNN})$ and recurrent neural networks $(\mathrm{RNN})$. Both have enough accuracy and are subject to composition. However, RNN is vulnerable to feature sequences, which can result in decreased correctness. Previous studies using a network similar to $\mathrm{CNN}$, namely $\mathrm{DBN}$, for the classification of slow cortical potentials in stroke [14]. CNN is widely used for image processing. An EEG signal can be viewed as an image, so its processing uses two-dimensional CNN, like previous studies for the detection of epileptic attacks [17]. However, not a few are also used for signal processing using one dimension. Some researchers used to identify EEG signals between stroke patients and healthy people [18], ischemic detection [19]. Another study to analyze epileptiform spikes on EEG signals [17] and motor imagery (MI) detection [20]. After undergoing rehabilitation, the post-stroke patient is expected to be healthy or have no stroke. NIHSS scales can be the standard for determining stroke levels from several indicators. But for the "minor" and "no stroke" levels, there is a slight difference. Therefore, we need another instrument, such as the EEG. This study proposed the CNN method for classifying EEG signals against post-stroke patients into both classes. The computational model of the EEG signal is built through wavelet extraction to get the right frequency range. EEG signals were extracted classified using CNN.

\section{RESEARCH METHOD}

The EEG signal classification of post-stroke patients used CNN, as shown in Figure 1, to identify "minor stroke," and "no stroke" class.

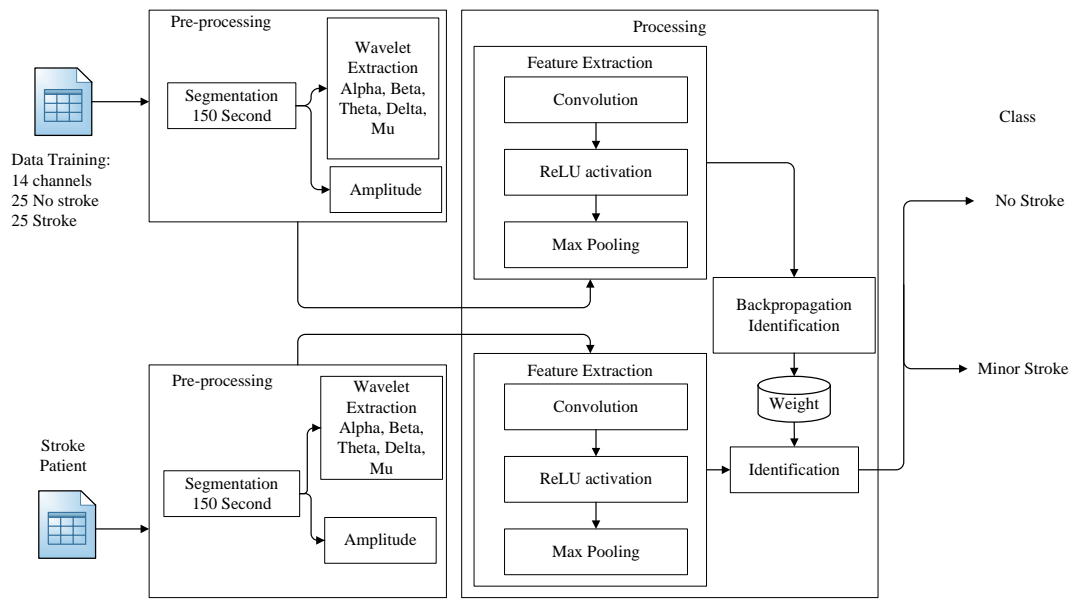

Figure 1. Identification of EEG signal of the post-stroke patient model 


\subsection{Data acquisition}

EEG signal data is recorded using Emotiv Epoch+ with a sampling frequency of $128 \mathrm{~Hz}$ from 14 channels, i.e., AF3, F7, F3, FC5, T7, P7, O1, O2, P8, T8, FC6, F4, F8. As subjects were 50 outpatients in the neurology clinic of RSIB hospital [8]. As a control group of the "No Stroke" class, recorded EEG signals from 25 healthy volunteers. Control groups are also used to identify strokes using portable EEG [16] and identified ischemic stroke [19]. At the rate $80 \%$ of all subjects as training data and $20 \%$ as non-training data. Each patient follows the instruction in Figure 2 to generate the appropriate waves. From 180 seconds of recording, the data used is only 120 seconds by removing 30 seconds beginning and ending.

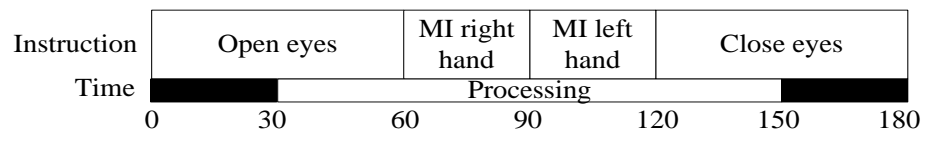

Figure 2. Instruction of data acquisition

\subsection{Wavelet extraction}

Wavelet transformation aims to split signals in two frequency ranges, approximation and detail or as frequency filter. The method is appropriate for non-stationary signals such as EEG [21]. Basis function $\Phi(\mathrm{n})$ called the mother wavelet as (1);

$$
\Phi_{j, k}(n)=2^{j / 2} \Phi\left(2^{j} n-k\right)
$$

where $\mathrm{j}$ and $\mathrm{k}$ are an integer that indicates the scaling and dilate of the basis function. It depends on the shape or position of the signal. $\Phi(\mathrm{n})$ is the wavelet family.

Signals with a $128 \mathrm{~Hz}$ sampling frequency may have a frequency component of 1-64 Hz or half. Each decomposition will be the half frequency signal as an approximation and a detail component. If it is repeated several steps, it can be extracted from Alpha or Mu waves, Theta, Delta wave, as in Figure 3.

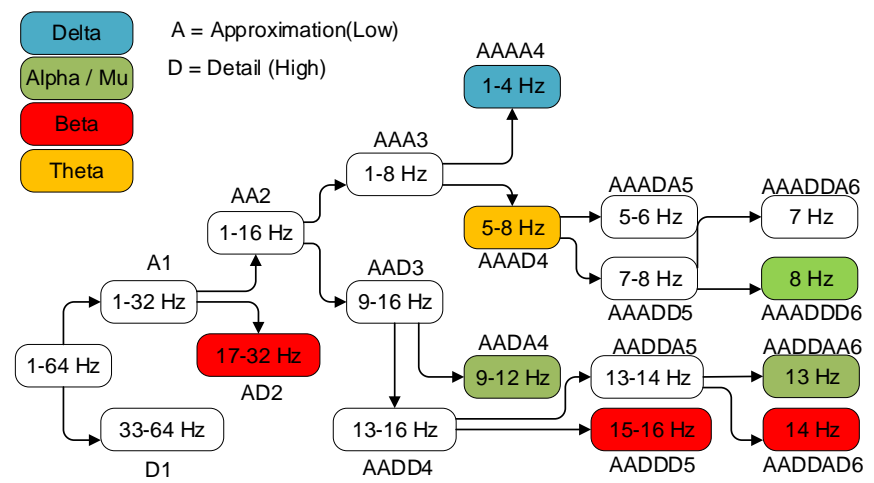

Figure 3. Wavelet decomposition

Discrete wavelet transformation passes a high-pass filter (detail), and a low-pass filter (approximation) signals shown in (2) and (3).

$$
\begin{aligned}
& \text { Approximation }=y_{\text {low }}(K)=\sum_{n} x(n) \cdot g(n-k) \\
& \text { Detail } \quad=y_{\text {high }}(K)=\sum_{n} x(n) \cdot g(n-k)
\end{aligned}
$$

Where $\mathrm{j}$ and $\mathrm{k}$ are an integer that indicates the scaling and dilate of the basis function. It depends on the shape or position of the signal. $\Phi(\mathrm{n})$ is the wavelet family. The feature extraction of the EEG signal is obtained by convolution, which also decomposes the signal to several levels. A previous study used Symlet 2 to eliminate noise and get the desired signal that is Gamma, Beta, Alpha, Theta, and Mu wave [8]. EEG signal wave extraction can use fast Fourier transform (FFT) or wavelet transformation. Wavelet capability is better for non-stationary signals such as EEG signals [22]. Wavelet extraction helps distinguish EEG signals, so that provide good accuracy [21]. 


\subsection{Convolution neural network}

CNN has the potential to process data in the time domain, such as an EEG signal [17]. In pattern recognition, what is very important is the extraction of EEG signals into features that match the classification variable. Some studies related to stroke using Alpha, Beta, Theta, and Delta waves [6, 20], other studies using brain symmetric index and relative frequency band ratio [23], frequency bands [24] and their comparisons [25], amplitude, rhythm [24] and MI [26]. This research used Delta, Theta, Alpha, Beta waves, MI, and amplitude based on a previous study [8]. Refer to Figure 2; the first 30 seconds during eye-opening contain Delta, Theta, Alpha, and Beta waves (1-32 Hz). As Figure 3, there are 64 points every second. Meanwhile, when the MI relation to $\mathrm{Mu}$ wave, that is 720 points. While the last 30 seconds raises the Alpha wave, which is 720 seconds. By adding the amplitude feature, the configuration as in Table 1.

Amplitude takes average each 0.0625 -second segment or 8 points per second. In Table 1, all features are on each channel, except for the Mu wave feature. So specifically, FC5 and FC6 channels have 3.960 points, while another channel has 3240 points. So there are 46.800 features. All features will normalize to reduce bias and get better learning [27]. In this study, normalization was carried out in a range of numbers between -1 and 1 to equalize the maximum and minimum values of each channel.

Table 1. CNN feature

\begin{tabular}{cclcl}
\hline No & Time (second) & \multicolumn{1}{c}{ Component } & Number of points & \multicolumn{1}{c}{ Description } \\
\hline 1 & 30 & Filter $(1-32 \mathrm{~Hz})$ & 1.920 & All channel \\
2 & 30 & Mu $(8-13 \mathrm{~Hz})$ & 360 & FC5 and FC6 only \\
3 & 30 & Mu $(8-13 \mathrm{~Hz})$ & 360 & FC5 and FC6 only \\
4 & 30 & Alpha $(8-13 \mathrm{~Hz})$ & 360 & All channel \\
5 & 120 & Average Amplitude & 960 & All channel \\
Total & & & 3.960 & \\
\hline
\end{tabular}

CNN consists of a convolution layer for extracting features and classification layers for learning, as in Figure 4. Usually, the classification or identification layer uses the multi-layer perceptron (MLP) architecture. In previous studies, CNN was used to identify ischemic stroke patients and no stroke people throughout looking at EEG signals into one-dimensional objects $[17,18]$. While in other studies, CNN was used to identify MI [28], and ECG [29]. The feature generated by this layer will be input into the CNN identification layer through training first. In learning, there are several ways to correct weights, such as stochastic gradient descent (SGD) or adaptive moment estimation (Adam). AdaDelta has a way of working similar to adaptive backpropagation, which has been used for BCI [30].

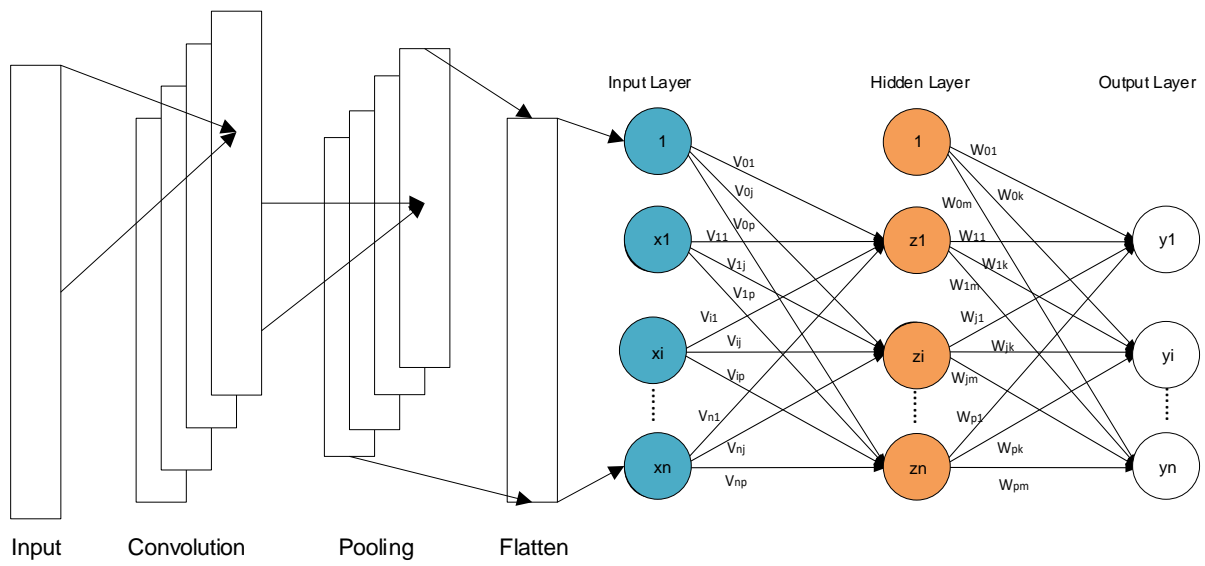

Figure 4. Convolutional neural networks architecture

\subsubsection{Feature extraction layer}

The layers contained in feature extraction are useful for translating input into a feature based on the data in the form of a vector. This feature extraction layer consists of a convolution layer and a sub sampling (pooling) layer. The hyper parameters of the convolution are depth, stride, and zero-padding, as shown in (4). The output of this layer is used to the next process, namely the subsampling process of each $\mathrm{m}_{1}$ map of the matrix with the size of $\mathrm{m} x \mathrm{n}$ and kernel function $\mathrm{C}$ calculated in rows $\mathrm{k}$ and column $\mathrm{y}$ in the first convolution layer. 


$$
\begin{aligned}
& F M_{\left(i_{l}, j_{l}\right)}^{\left(l, m_{l}\right)}=f\left(\sum_{r_{l}=0}^{k_{h-1}} \sum_{c_{l}=0}^{k_{w-1}} C_{\left(r_{l}, c_{l}\right)}^{\left(l, m_{l}\right)} * F M_{\left(r_{l}+i_{l-1}, c_{l}+j_{l-1}\right)}^{(l-1)}\right) \\
& F(x)=\max (0, x)
\end{aligned}
$$

Where $K_{h}$ is height, and $K_{w}$ is the length of the kernel. Then $r_{l}$ and $c_{l}$ are the length and height of the kernel layer index 1 , and $\mathrm{C}$ is the convolution kernel. After the convolution process, then the next is the activation process using the rectified linear units (ReLU), which is useful for normalizing all negative values to zero using (5).

Next, the downsampling or pooling layer serves to reduce the number of parameters from the feature map that will be used in the classification layer. Also, pooling speeds up computing and controls overfitting. Downsampling is accomplished by getting the value of a feature map with a determined size and stride. This study used Max pooling, which only regards the highest value in the feature map.

\subsubsection{Identification layer}

The second layer of CNN is an identification layer composed of several layers. Each layer is formed of fully connected neurons with other layers, known as multi-layer perceptron. Meanwhile, the learning process used some methods such as Backpropagation [28]. This layer handles the dropout process to overcome overfitting in training, which can cause a reduction [31]. Dropout works by temporarily removing a neuron in the form of hidden layers, input layers, or output layers that are in the network. The activation function, usually using Softmax that convert the output into a probability for each class, as shown in (6).

$$
y_{j}=\frac{e^{x^{T} w_{j}}}{\sum_{k=1}^{K} e^{x^{T} w_{k}}}
$$

A loss function is used to measure the error between expectations of the target class compared to the actual data from the computational results. The loss function that can be used is Cross-Entropy (7). Where Loss is the distance, $\mathrm{S}$ is the result of the Softmax S, and L is the class label.

$$
\operatorname{Loss}(S, L)=-\sum_{i} L_{i} \log \left(S_{i}\right)
$$

\section{RESULTS AND DISCUSSION}

EEG signals of post-stroke patients can be recognized by observing wave density or rhythm, amplitude and also differences in the magnitude of channel pair, and the presence of Alpha, Beta, Delta, and Theta waves. Therefore, learning features used Alpha, Beta, Delta, Teta wave, and amplitude. The key to the successful classification of EEG signals is extraction into frequency components. Therefore, Wavelet performance needs to be tested first.

\subsection{Wavelet extraction}

Wavelet extraction using Symlet 2 at $0.5-32 \mathrm{~Hz}$ is illustrated in Figure 5. The blue line is the original signal, and the orange line is the result of a signal that has performed wavelet extraction. It can be seen that a low wave signal is obtained by decomposing to eliminate noise in the EEG signal via wavelet extraction.

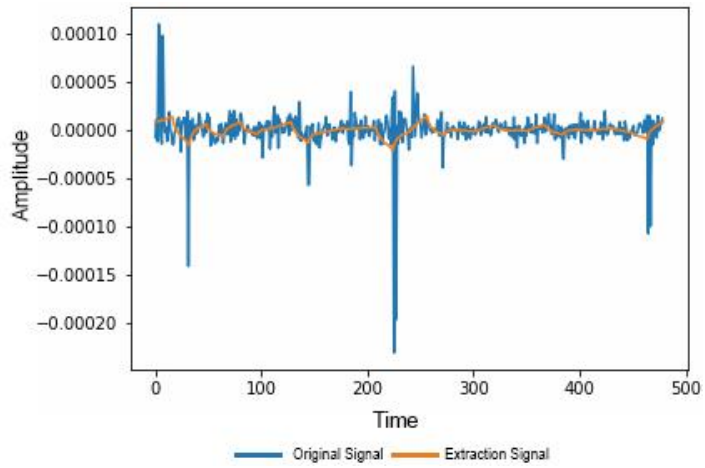

Figure 5. Comparison between original signals with wavelet extraction 


\subsection{Compared between optimization model}

The classification that is performed using deep learning with the CNN model needs configuration to obtain high accuracy. In this study, using VGG19 architecture as a learning process. The architecture has 16 convolution layers and five pooling layers. A suitable configuration is required to update the weights during the training so that they get maximum results. The weight correction can be achieved with various optimizers. Some of them are adaptive moment estimation (Adam) and stochastic gradient descent (SGD). This research optimizes the learning rate of 0.01 and 500 epochs. Accuracy and Loss value each optimizer are shown in Figure 6, Figure 7, and Table 2. The Adam model (orange color) was more stable than the SGD model (red color) for new data (valid) in the small epoch. But SGD has higher accuracy compared to Adam. Because SGD still changes the value of accuracy to find the best value compared to Adam [32]. The convergence of the Adam model to SGD is seen in Loss in Figure 7, so both values are consistent. Table 2 shows the optimization of SGD is better than Adam, although the loss value is higher. But it is not significant.

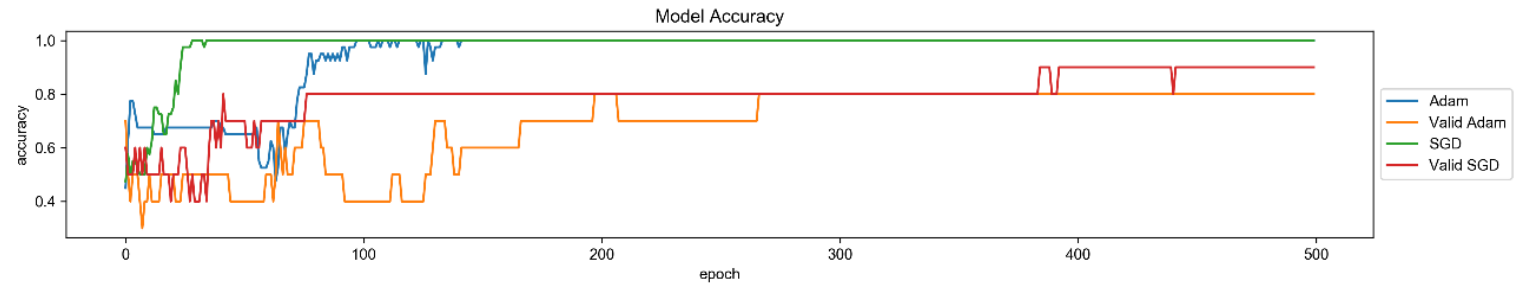

Figure 6. Accuracy of Adam and SGD model

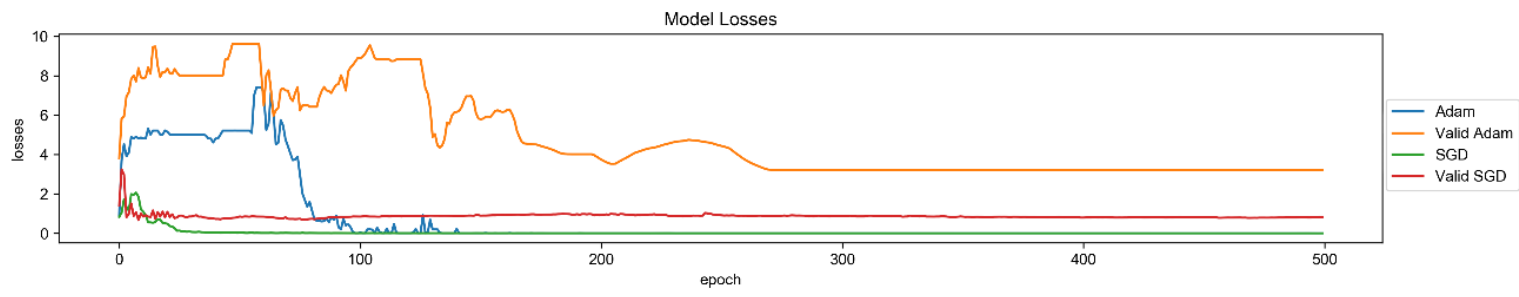

Figure 7. Loss of Adam and SGD model

Besides, to accuracy, testing is performed on computational time in learning. This study carried out learning in 500 epochs with GPU Nvidia Geforce GTX 1050 4GB. A comparison of computing time between SGD and Adam models is shown in Table 2. The absence of Beta or amplitude waves, even though they provide low accuracy, are obtained with a shorter time. Based on Table 1, the number of amplitude points and Beta waves is the same, which gives the corresponding computational time reduction as Table 2.

Table 2. Compare Adam and SGD model

\begin{tabular}{llllllll}
\hline \multicolumn{2}{l}{$\begin{array}{l}\text { Configuration } \\
\text { Amplitude }\end{array}$} & Beta & Model & Training data & & New data \\
Accuracy(\%) & Loss & Accuracy $(\%)$ & Loss & Computation time (sec) \\
\hline$\checkmark$ & $\checkmark$ & Adam & 100 & 0.0000 & 80.00 & 3.7571 & 180 \\
& & SGD & 100 & 0.0007 & 90.00 & 0.8221 & 160 \\
$\times$ & $\checkmark$ & Adam & 100 & 0.0000 & 60.00 & 6.4120 & 140 \\
& & SGD & 100 & 0.0009 & 70.00 & 1.9362 & 120 \\
$\checkmark$ & $\times$ & Adam & 100 & 0.0000 & 50.00 & 5.3427 & 140 \\
& & SGD & 100 & 0.0007 & 70.00 & 1.0861 & 120 \\
\hline
\end{tabular}

\subsection{Performance of amplitude and Beta wave}

The experiment showed that involve amplitude as a learning feature gave more accurate for both models, as shown in Table 2. From Figure 8, it can be seen that the accuracy of SGD is also higher than Adam. And Adam model is more stable too than SGD for configurations without amplitude. CNN works based on the convolution of time series data with the kernel without affording a direct connection between sequential data. Therefore, the use of wave features and amplitude still provides higher accuracy. This experiment is consistent with previous research [8]. 


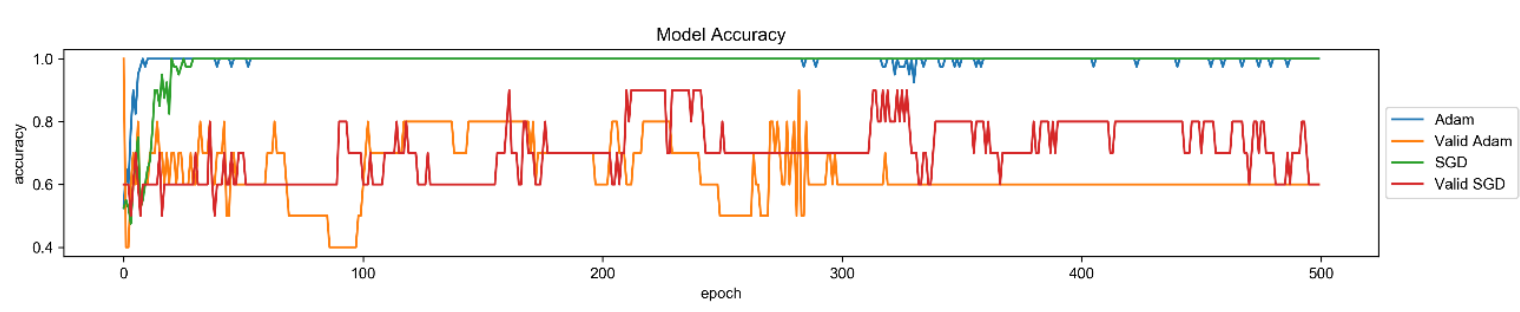

Figure 8. Accuracy of Adam and SGD without amplitude

Although the presence of low-frequency wave dominance characterizes strokes, Beta waves also contribute to MI in addition to $\mathrm{Mu}$ waves [26]. Therefore its appearance is also significant for increasing accuracy. The result showed that the accuracy of the SGD model is higher too than the Adam, without the presence of Beta waves model, as shown in Figure 9.

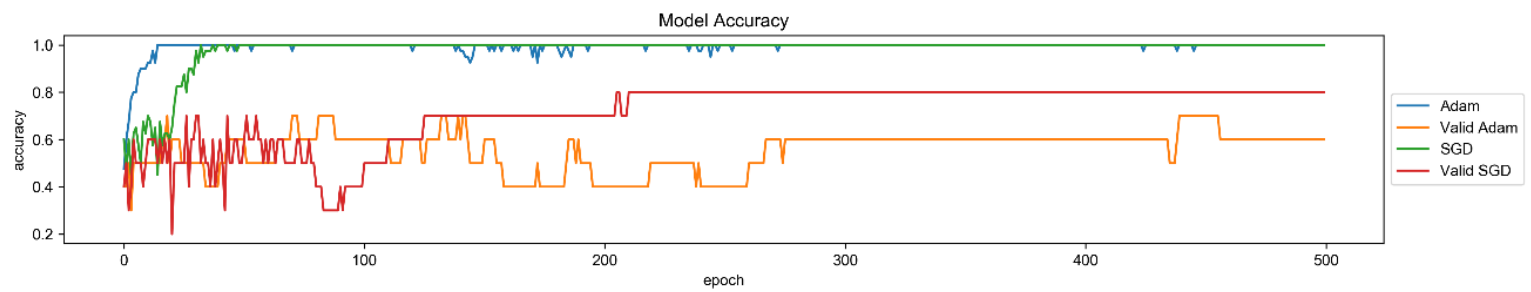

Figure 9. Accuracy of Adam and SGD without Beta wave

\section{CONCLUSION}

This study identified two levels of stroke from NIHSS using wavelet and CNN with training data accuracy of $100 \%$ and non-training data at $90 \%$. The essential thing in identification is the proper configuration of input features according to the identified variable, which is stroke. Using low waves (Delta and Theta), Alpha, motor imagery (Beta and $\mathrm{Mu}$ ), and amplitude are significant in the classification of "minor strokes" to "no strokes", thus providing good accuracy. Identification using CNN also needs to pay attention to optimization models that provide high correctness and stability. Sometimes, both of them are contradictive, so accuracy considerations are preferred. The SGD model is more oscillating at the beginning of the epoch but provided better accuracy than the Adam model.

In the future, we need to find the configuration of the channel. Appropriate channel processing can increase accuracy and reduce computing time that is more realistic to implement. Also worth noting is the setting and sequence of features that provide high accuracy and relatively low computing time.

\section{ACKNOWLEDGEMENTS}

The research was funded by PDUPT Kemenristekdikti. Thanks also to Nerve Clinic and Rehabilitation, Al Islam Hospital Bandung, for the assist of recording EEG signals from post-stroke patients

\section{REFERENCES}

[1] World Health Organization, "Global health estimate," 2012.

[2] P. Lyden, "Using the national institutes of health stroke scale: A cautionary tale," Stroke, vol. 48, no. 2, pp. 513-519, 2017.

[3] S. P. Finnigan, S. E. Rose, M. Walsh, M. Griffin, A.L. Janke, K. L. McMahon, R. Gillies, M. W. Strudwick, C. M. Pettigrew, J. Semple, J. Brown, P. Brown, and J. B Chalk, "Correlation of quantitative EEG in acute ischemic stroke comparison with 30-day NIHSS score: Comparison with diffusion and perfusion MRI," Stroke, vol. 35, no. 4, pp. 899-903, 2004.

[4] L. Chen, P. Bentley, and D. Rueckert, "Fully automatic acute ischemic lesion segmentation in DWI using convolutional neural networks," NeuroImage: Clinical, vol. 15, no. June, pp. 633-643, 2017

[5] G. Bartur, K. Joubran, S. Peleg-Shani, J-J. Vatine, and G. Shahaf, "An EEG tool for monitoring patient engagement during stroke rehabilitation : A feasibility study,” BioMed Research International, vol. 2017, pp. 1-11, 2017.

[6] C. Bentes, A. R. Peralta, P. Viana, H. Martins, C. Morgados, C. Casimiro, A. C. Franco, A. C. Fonseca, 
R. Geraldes, P. Canhao, T. P. e Melo, T. Pavia, and J. M. Ferro, "Clinical neurophysiology quantitative EEG and functional outcome following acute ischemic stroke," Clinical Neurophysiology, vol. 129, no. 8, pp. 1680-1687, 2018.

[7] K. K. Ang, C. Guang, K. S. G. Chua, B. T. Ang, C. W. K. Kuah, C. Wang, K. S. Phua, Z. Y. Chin, and H. Zhang, "A large clinical study on the ability of stroke patients to use an EEG-based motor imagery brain-computer interface," Clinical EEG and Neuroscience, vol. 42, no. 4, pp. 253-258, 2011.

[8] E. C. Djamal, D. P. Gustiawan, and D. Djajasasmita, "Significant variables extraction of post-stroke EEG signal using wavelet and SOM kohonen," TELKOMNIKA (Telecommunication Computing Electronics and Control), vol. 17, no. 3, pp. 1149-1158, 2019.

[9] A. Khosla, P. Khandnor, and T. Chand, "A comparative analysis of signal processing and classification methods for different applications based on EEG signals," Biocybernetics and Biomedical Engineering, vol. 40, no. 2, pp. 649-690, 2020.

[10] E. C. Djamal and Suprijanto, "Recognition of electroencephalogram signal pattern against sound stimulation using spectral of wavelet," TENCON, pp. 374-378, 2011.

[11] S. Z. Bong, K. Wan, M. Murugappan, N. M. Ibrahim, Y. Rajamanickam, and K. Mohamad, "Biomedical signal processing and control implementation of wavelet packet transform and non linear analysis for emotion classification in stroke patient using brain signals," Biomedical Signal Processing and Control, vol. 36, pp. 102-112, 2017.

[12] Z. Mohammadi, J. Frounchi, and M. Amiri, "Wavelet-based emotion recognition system using EEG signal," Neural Computing and Applications, vol. 28, no. 8, pp. 1985-1990, 2017.

[13] N. Singh, M. Saini, N. Kumar, K. K. Deepak, S. Anand, M. V. P. Srivastava, and A. Mehndiratta, "Time-frequency analysis of motor-evoked potential in patients with stroke vs healthy subjects : A transcranial magnetic stimulation study," Comprehensive Clinical Medicine, vol. 1, no. 10, pp. 764-780, 2019.

[14] G. Altan, Y. Kutlu, and N. AllahverdI, "Deep belief networks based brain activity classification using EEG from slow cortical potentials in stroke," International Journal of Applied Mathematics, Electronics and Computers, no. 4, pp. 205-210, 2016.

[15] M. Murugappan, N. Ramachandran, and Y. Sazali, "Classification of human emotion from EEG using discrete wavelet transform," J. of Biomedical Science and Engineering, vol. 3, no. 4, pp. 390-396, 2010.

[16] M. Gottlibe, O. Rosen, B. Weller, A. Mahageny, N. Omar, A. Khuri, I. Srugo, and J. Genizi, "Stroke identification using a portable EEG device-A pilot study," Neurophysiologie Clinique, vol. 50, no. 1, pp. 21-25, 2020.

[17] M. Zhou, C Tian, R. Cao, B. Wang, Y. Niu, T. Hu, H. Guo, and J. Xiang,, "Epileptic seizure detection based on EEG signals and CNN," Front. In Neuroinformatics, vol. 12, no., pp. 1-14, 2018.

[18] E. C. Djamal, W. I. Furi, and F. Nugraha, "Detection of EEG signal post-stroke using FFT and convolutional neural network," 6th International Conf. on Electrical Engineering, Computer Science and Informatics (EECSI 2019) pp. 18-23, 2019.

[19] E. P. Giri, M. I. Fanany, A. M. Arymurthy, and S. K. Wijaya, "Ischemic stroke identification based on EEG and EOG using ID convolutional neural network and batch normalization," International Conference on Advanced Computer Science and Information Systems, ICACSIS 2016, pp. 484-491, 2017.

[20] S. U. Amin, M. Alsulaiman, G. Muhammad, M. A. Mekhtiche, and M. S. Hossain, "Deep learning for EEG motor imagery classification based on multi-layer CNNs feature fusion," Future Generation Computer Systems, vol. 101, pp. 542-554, 2019.

[21] H. Göksu, "BCI oriented EEG analysis using log energy entropy of wavelet packets," Biomedical Signal Processing and Control, vol. 44, pp. 101-109, 2018.

[22] S. Z. Bong, K. Wan, M. Murugappan, N. M. Ibrahim, Y. Rajamanickam, and K. Mohamad, "Implementation of wavelet packet transform and non linear analysis for emotion classification in stroke patient using brain signals," Biomedical Signal Processing and Control, vol. 36, pp. 102-112, 2017.

[23] W. R. W. Omar, R. Jailani, M. N. Taib, R. M. Isa and Z. Sharif, "Assessment of acute ischemic stroke brainwave using relative power ratio," in 2013 IEEE 9th International Colloquium on Signal Processing and its Applications, pp. 310-313, 2013.

[24] F. Vecchio, P. Caliandro, G. Reale, F. Miraglia, F. Piludu, G. Masi, C. Lacovelli, C. Simbolotti, L. Padua, E. Leone, F. Alu, C. Colosimo, and P. M. Rossini, "Clinical neurophysiology acute cerebellar stroke and middle cerebral artery stroke exert distinctive modifications on functional cortical connectivity : A comparative study via EEG graph theory," Clinical Neurophysiology, vol. 130, no. 6, pp. 997-1007, 2019.

[25] N. Wang, Y. Pu, Y. Li, J. Tian, D. Liu, Z. Zhang, Q. Sheb, L. Liu, and W. Sun, "Characterization of electroencephalography of strokes based on time-frequency analysis," Computer Aided Chemical Engineering, vol. 44, pp. 2161-2166, 2018.

[26] S. Shahid, R. Sinha, and G. Prasad, "Mu and Beta rhythm modulations in motor imagery related post-stroke EEG: A study under BCI framework for post-stroke rehabilitation," Bio Med Central Neurosci., vol. 11, SI, pp. 1-2, 2011.

[27] M. M. Shaker, "EEG waves classifier using wavelet transform and Fourier transform," International Journal of Biomedical Science, vol. 1, pp. 85-90, 2007.

[28] M. Dai, D. Zheng, R. Na, S. Wang, and S. Zhang, "EEG classification of motor imagery using a novel deep learning framework," Sensor, vol. 19, no. 3, pp. 1-16, 2019.

[29] Q. Zhang, D. Zhou, and S. Member, "HeartID : A multiresolution convolutional neural network for ECG-based biometric human identification in smart health applications," IEEE Access, vol. 5, pp. 11805-11816, 2017.

[30] E. C. Djamal, Suprijanto, and S. J. Setiadi, "Classification of EEG-based hand grasping imagination using autoregressive and neural networks," Jurnal Teknologi, vol. 78, no. 6-6, pp. 105-110, 2016.

[31] A. Krizhevsky, I. Sutskever, and G. E. Hinton, "ImageNet classification with deep convolutional neural networks," 
Advances in Neural Information Processing Systems, pp. 1097-1105, 2012.

[32] A. C. Wilson, R. Roelofs, M. Stern, N. Srebro, and B. Recht, "The marginal value of adaptive gradient methods in machine learning," Advances in Neural Information Processing Systems, pp. 4148-4158, 2017.

\section{BIOGRAPHIES OF AUTHORS}

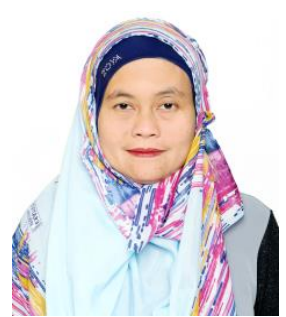

Esmeralda Contessa Djamal received a Bachelor's degree in Engineering Physics from Institut Teknologi Bandung in 1994, a Master's degree in Instrument and Control from Institut Teknologi Bandung in 1998. Since Ph.D. dissertation until now, research on EEG classification and finished doctoral program from Institut Teknologi Bandung in 2005. She is a lecturer of Informatics Department, Universitas Jenderal Achmad Yani.

E-mail: esmeralda.contessa@lecture.unjani.ac.id

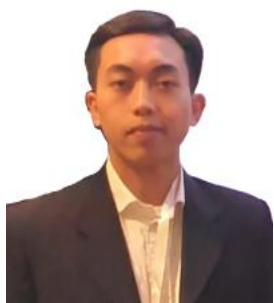

Rizkia Ilham Ramadhan received his bachelor's degree in Informatics department from Universitas Jenderal Achmad Yani in 2019. E-mail: rizkiailham.r@gmail.com

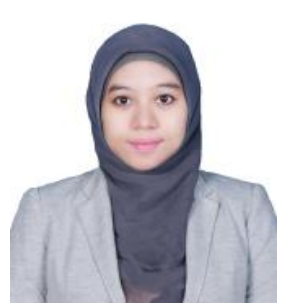

Miranti Indar Mandasari, received a Bachelor's degree in Engineering Physics from Institut Teknologi Bandung in 2008, Master's degree in Instrument and Control from Institut Teknologi Bandung in 2020, and a Ph.D. degree from Centre for Language \& Speech Technology Radboud University Nijmegen. She is a lecturer of Engineering physics Department, Institut Teknologi Bandung. E-mail: miranti.indar.mandasari@gmail.com

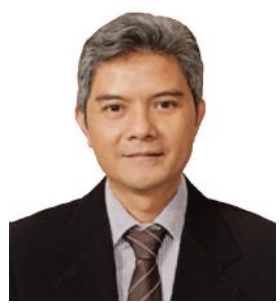

Daswara Djajasasmita is a neurologist in the Rumah Sakit Islam Bandung. He completed his medical education from Universitas Padjadjaran in 1992 and a Neurology specialist from Universitas Padjajaran in 2006. Until now he is also a lecturer at the Faculty of Medicine, Universitas Jenderal Achmad Yani. E-mail: daswaradj@yahoo.com 